\title{
Biomimic Enamel Remineralization by Hybridization Calcium- and Phosphate-Loaded Liposomes with Amelogenin-Inspired Peptide
}

\author{
Jingjing Luo ${ }^{1, a}$, Tianyun $\mathrm{Ning}^{1}$,Ying $\mathrm{Cao}^{1}$, Xiaopeng $\mathrm{Zhu}^{1}$, Xiaohua $\mathrm{Xu}^{1}$, \\ Xuyan Tang ${ }^{1}$,Chun Hung $\mathrm{Chu}^{2, \mathrm{~b}}$, Quanli $\mathrm{Li}^{1, \mathrm{c}}$ \\ ${ }^{1}$ Key Lab. of Oral Disease Research of Anhui Province, College of Stomatology, Anhui Medical \\ University, Hefei, China \\ ${ }^{2}$ Faculty of Dentistry, The University of Hong Kong, Hong Kong, China \\ a luojing8511@163.com, ${ }^{b}$ chchu@hku.hk, ${ }^{c}$ ql-li@126.com ( corresponding author)
}

Keywords: Enamel, Biomimetic mineralization, Temperature-sensitive liposomes, Amelogenin, Self-assembly

\begin{abstract}
We here report a novel biomimetic mineralization strategy for enamel remineralization by intergration of calcium phosphate loaded and thermally triggered liposomes and a self-assembly amelogenin-inspired peptide. Firstly, calcium and phosphate loaded temperature sensitive liposomes were synthesized by Interdigitation-fusion method with 1,2-bis(palmitoyl)-sn-glycero-3-phosphorcholine (DPPC) and 1,2-bis(myristoyl)-sn-glycero- 3-phosphocholine (DMPC) at mass ratio of 9:1. The liposomes were stable at room temperature, but slowly released calcium and phosphate ions if heated to $37{ }^{\circ} \mathrm{C}$. Secondly, a novel polyanion amelogenin-inspired oligopeptide (Gln-Pro-Ala) - $^{-}$ Thr-Lys-Arg-Glu-Glu-Val-Asp) was synthesized by standard solid-phase. Finally, the mixture of peptide and liposomes solution was exposed to enamel surface at $37{ }^{\circ} \mathrm{C}$. The results showed oriented enamel-like hydroxylapatite evenly deposited on enamel surface.
\end{abstract}

\section{Introduction}

Matured enamel is composed of $95 \mathrm{wt} \%$ nano rod-like hydroxyapatite (HA). The nano HA crystals have a cross-section of $25-100 \mathrm{~nm}$ and an undetermined length of $100 \mathrm{~nm}$ to $1000 \mathrm{~nm}$ or longer along the c-axis, and self-assemble into prism microstructure [1]. Biomineralization process is selfassembly and an organic-matrix-mediated biomineralization, where some types of organic macromolecules self-assemble and may be used as templates for controlling the nucleation and growth of mineral crystals to form hierarchical hybrid materials [2]. Recently, with the development of understanding the mechanisms of biomineralization, and nanomaterial fabrication technique, biomimetic mineralization strategy has been used to regenerate the hierarchical microstructure of the dental tissue [3-5].

During enamel formation, amelogenin, the major enamel protein constituting approximately $90 \%$ of all organic matrix material, spontaneously self-assembles into nanospherical supermolecular structures. This supermolecular structure plays a vital role in HA nucleation and growth, and furtherly directs the HA crystals to form a well-organized prism pattern [6]. The molecular mechanism of amelogenin self-assembly is that its primary structure preserves a bipolar nature, especially proline-rich sequences that contain a repetitive sequence of (Gln-Pro-X)n forms with a $\beta$ spiral secondary structure showing a unique globular monomers, and the hydrophilic flexible C-terminal (-Thr-Lys-Arg-Glu-Glu-Val-Asp) "tail" which interacts with calcium and initializes HA nucleation exposes on the surface of the globular monomers [7]. Thus, we designed a novel amelogenin-like oligopeptide ( (Gln-Pro-X)n- Thr-Lys-Arg-Glu-Glu-Val-Asp) to control enamel remineralization.

On the other hand, a biological strategy during mineralized tissue formation is the use of matrix vesicle (lipid vesicle compartments) to sequester calcium and phosphate ions, control ion transport, and to control mineral particles deposited within developing tissues[8, 9]. Messersmith have 
developed a temperature-sensitive liposomes system to mimetic matrix vesicle to control mineralization study $[10,11]$. The liposomes loaded calcium and phosphate salts, and were stable at room temperature, but slowly released calcium and phosphate ions when heated to $37^{\circ} \mathrm{C}$.

In the present study, We intergrated the calcium phosphate loaded liposomes and the self-assembly amelogenin-inspired peptide to build a novel biomimetic mineralization strategy for enamel remineralization.

\section{Experimental}

Calcium- and phosphate-loaded liposomes preparation Calcium- and phosphate-loaded liposomes were prepared using the interdigitation-fusion (IF) method as reported by Messersmith with some modification [10,11]. Briefly, 1,2-bis(palmitoyl)-sn-glycero-3-phosphocholine (DPPC > 99\%) and 1,2-bis(myristoyl)-sn- glycero-3-phosphocholine (DMPC $>99 \%$ ) (Sigma-Aldrich Trading Co., Ltd, American)were prepared at the mass ratio of 9:1, and hydrated with aqueous $\mathrm{CaCl}_{2}$ or $\mathrm{Na}_{2} \mathrm{HPO}_{4}$. Then, the resultant suspensions were probe-sonicated and centrifuged. Absolute ethanol was added to the liposome suspensions. The samples were sealed and incubated for $15 \mathrm{~min}$ at room temperature followed by incubated for $15 \mathrm{~min}$ at $50{ }^{\circ} \mathrm{C}$. Then allow ethanol evaporation resulting in $\mathrm{Ca}^{2+}$ and $\mathrm{PO}_{4}{ }^{3-}$-loaded vesicles. Vesicle suspensions were then combined with an equal volume of buffered $\mathrm{NaCl}$ solution, vortexed, and centrifuged at $16,000 \mathrm{~g}$ for $5 \mathrm{~min}$. This process was repeated until $\mathrm{Ca}^{2+}$ (or $\mathrm{PO}_{4}{ }^{3-}$ ) could not be detected in the supernatant using a colorimetric test with AIII dye. The liposomes were characterized with SEM,TEM and Dynamic light scattering(DLS).

Prepared of the amelogenin-like oligopeptide The amelogenin-like oligopeptide of (Gln-ProAla) ${ }_{4}$-Thr-Lys-Arg-Glu-Glu-Val-Asp was synthesized by standard solid-phase peptide synthesis. The HPLC-purified peptide was checked by analytical HPLC and mass spectrometry.

Biomimetic remineralization of etched enamel surface $1 \mathrm{~mm}$ thick slabs of human enamel were etched with $37 \%$ phosphoric acid. $\mathrm{Ca}^{2+}$ and $\mathrm{PO}_{4}{ }^{3-}$-loaded liposomes were diluted with $50 \mathrm{mM}$ HEPES at the ratio of about 1.7:1. PA solution were prepared and dissolved in deionized water at a concentration $20 \%$ by weight. The solution was adjusted to a $\mathrm{pH}$ of 9.0 using $1 \mathrm{M} \mathrm{NaOH}$. Lastly, the mixture of peptide and liposomes solution was exposed to enamel surface at $37^{\circ} \mathrm{C}$ in oven for two weeks. The enamel surface were characterized with SEM, DR-FTIR and film-XRD.

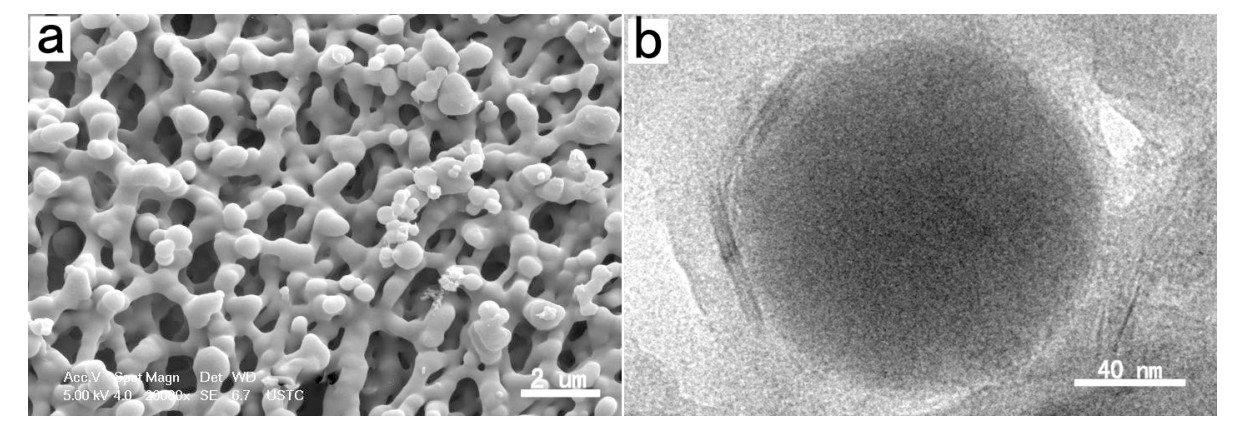

Fig. 1 a) SEM images of calcium -loaded temperature-sensitive liposomes; b) images of the liposomes of TEM

\section{Results and Discussion}

Liposomal synthesis and thermally triggered $\mathrm{Ca}^{2+}$ and $\mathrm{PO}_{4}{ }^{3-}$ release $\mathrm{We}$ prepared the temperature-sensitive liposomes by the interdigitation-fusion method (IF) as reported by Messersmith with some modification. The important factor to induce interdigitatio is the concentrations of ethanol. This method formed large unilamellar liposomes with a high encapsulation efficiency [12]. The encapsulation efficiency of Ca-loaded vesicles was $70 \sim 85 \%$, whereas the encapsulation efficiency of $\mathrm{PO}_{4}{ }^{3-}$-loaded vesicle was $60 \sim 80 \%$. It is found that liposomes morphology was a uniform global particles with the diameters about $161 \mathrm{~nm}$ (Fig. 1). The size of the $\mathrm{Ca}^{2+}$ and $\mathrm{PO}_{4}{ }^{3-}$-loaded liposomes of 
size similar to matrix vesicles $[13,14]$. If the liposomal was exposed to $37^{\circ} \mathrm{C}$ enviroment, $\mathrm{Ca}^{2+}$ and $\mathrm{PO}_{4}{ }^{3-}$ could slowly released from the liposomes tested with AIII dye. Release of entrapped ions at this temperature was due to increased permeability of phospholipid bilayers at the lipid chain melting temperature. Messersmith et al have proved the diffusion of $\mathrm{Ca}^{2+}$ and $\mathrm{PO}_{4}{ }^{3-}$ out of liposomes and their reaction to form crystalline calcium phosphate minerals, which were deposited on dentin and enamel surfaces or formation of mineral/collagen composite $[11,12]$. In the present study, we integrated the liposomes and the self-assembly amelogenin-inspired peptide to for enamel remineralization.

Biomimetic mineralization of etched enamel It is found that the enamel surface only treated with liposomes suspension had a few amount of HA crystal deposition, and the precipitate distributed randomly. In contrast, the experimental groups treated with the mixture solution of liposomes and polyanion peptide showed that the rob-like crystals almost fully covered the etched enamel surface, and distributed evenly to make the surface smoothly (Fig. 2). The deposited crystals assembled along its c-axis.
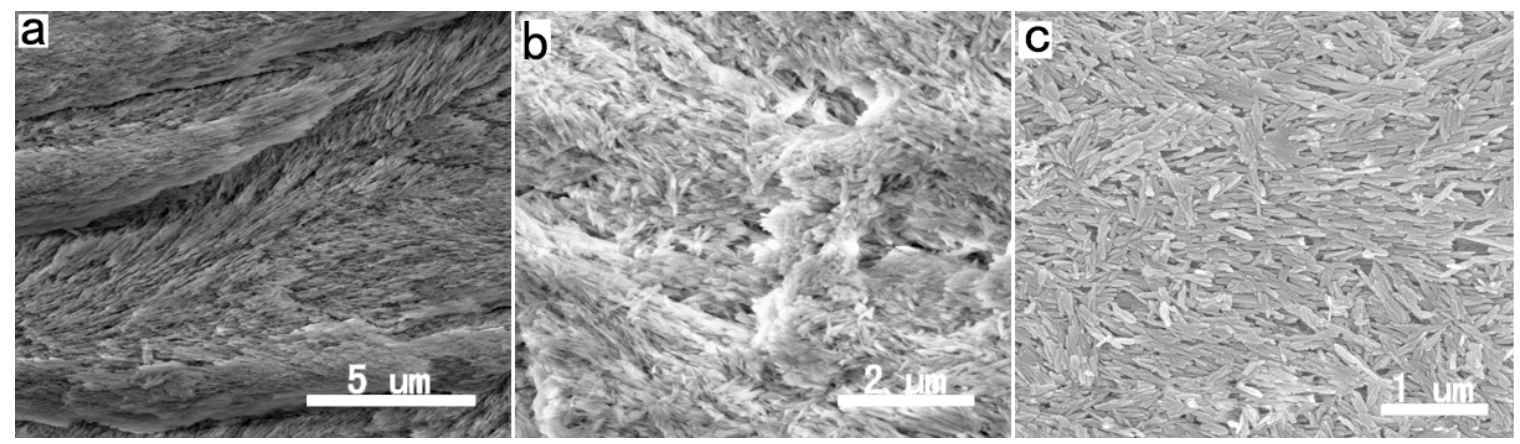

Fig. 2 SEM images of mineral crystals deposited on etched enamel surface. a) enamel etched with $37 \%$ phosphoric acid only; b) etched enamel surface treated with liposomes suspension; c) etched enamel surface treated with with liposome and polyanion peptide

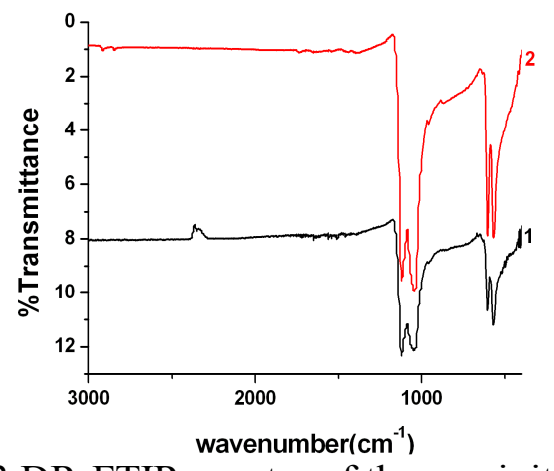

Fig. 3 DR-FTIR spectra of the precipitate, (1) control group (mineralization with liposome only); (2) experimental group(mineralization with liposome and polyanion peptide)

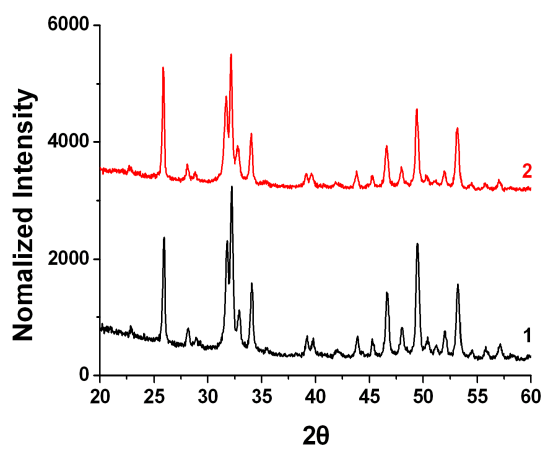

Fig. 4 X-ray diffraction pattern of the precipitate, (1) control group (mineralization with liposome only); (2) experimental group (mineralization with liposome and polyanion peptide)

In the FTIR spectrum of the treated enamel surface (Fig. 3), the peak at approximately $1100 \mathrm{~cm}^{-1}$, $1040 \mathrm{~cm}^{-1}, 650 \mathrm{~cm}^{-1}, 565 \mathrm{~cm}^{-1}$ represent $\mathrm{PO}_{4}{ }^{3-}$, respectively, but the intensity of the experiment groups was much more stronger than the control groups. All the XRD patterns of the control and experimental groups showed the characteristic peaks of hydroxyapatite. All the 002 lattice plane $\left(2 \theta=26^{\circ}\right)$ were enhanced, which suggested the HA crystal arranged along its c-axis. The orientation in experimental groups came from the coating HA, while the control groups came from the substrate enamel primes. 
From the above results, we found that the amelogenin-inspired oligopeptide polyanion peptide could induce enamel remineralization with high effectively, and could induce HA crystals to self-assemble into enamel like HA orientation. Thus, the novel amelogenin-inspired oligopeptide polyanion peptide may be a useful tool to be used in biomimetic mineralization study for enamel regeneration.

\section{Conclusions}

Temperature sensitive $\mathrm{Ca}^{2+}$ and $\mathrm{PO}_{4}{ }^{3-}$ loaded liposomes combining with self-assembly amelogenin-inspired oligopeptide polyanion peptide can effectively induce enamel remineralization. This biomimetic mineralization strategy provides a potential method mimicking the processes that occur during natural mineralized tissue formation.

\section{Acknowledgements}

This study was supported in part by the National Natural Science Foundation of China (NSFC) (Grant No.30973352 and 81070864), and the NSFC/RGC Joint Research Scheme (Grant No. 81061160511 and N_HKU 776/10).

\section{References}

[1] H.C. Margolis, E. Beniash,C.E. Fowler, Role of macromolecular assembly of enamel matrix proteins in enamel formation, J. Dent. Res.85( 2006) 775-793.

[2] S. Gajjeraman, K. Narayanan, J. Hao, C. Qin, George A, Matrix macromolecules in hard tissues ontrol the nucleation and hierarchical assembly of hydroxyapatite, J. Biol. Chem.282(2007) 1193-1204.

[3] J. Moradian-Oldak, The regeneration of tooth enamel, Dimens. Dent. Hyg.7( 2009)12-15.

[4] S. Busch, Regeneration of Human Tooth Enamel, Angew. Chem. Int. Ed. 43(2004) 1428 -1431.

[5] F.R. Tay, D.H. Pashley, Guided tissue remineralisation of partially demineralised human dentine,Biomaterials.29( 2008)1127-1137.

[6] J. Moradian-Oldakc, Amelogenins: Assembly, Processing and Control of Crystal Morphology, Matrix Biol. 20(2001) 293-305

[7] C. Du, G. Falini, S. Fermani, Supramolecular Assembly of Amelogenin Nanospheres into Birefringent Microribbons, Science. 307(2005)1450-1454.

[8] H.A. Lowenstam, S. Weiner, On Biomineralization, Oxford University Press, Oxford, 1989.

[9] S.Y. Ali, S.W. Sadjera, H.C. Anderson, Isolation and characterization of calcifying matrix vesicles from epiphyseal cartilage, Proc. Natl. Acad. Sci. 67 (1970) 1513.

[10] W.L. Murphy, P.B. Messersmith, Compartmental control of mineral formation: adaptation of a biomineralization strategy for biomedical use, Polyhedron .19(2000) 357-363.

[11] A.W. Pederson, J.W. Ruberti, P.B. Messersmith, Thermal assembly of a biomimetic mineral/collagen composite, Biomaterials.24 (2003) 4881-4890.

[12] P.L. Ahl, L. Chen, W.R. Perkins, S.R. Minchey, L.T. Boni, T.F.Taraschi, A.S. Janoff, Interdigitation-fusion: a new method for producing lipid vesicles of high internal volume, Biochim. Biophys. Acta. 1195 (1994) 237-244.

[13] E.D. Eanes, A.W. Hailer, J.L. Costa, Calcium phosphate formation in aqueous suspensions of multilamellar liposomes, Calcif. Tissue Int. 36 (1984)421-430.

[14] E.D. Eanes, Calcium phosphate formation in aqueous suspensions of anionic liposomes, Bone. 6 (1985) 474-475. 